\title{
Scrotum Hernia with Large Volume of Ascites
}

Zhong Jia*, Yu Zhou, Siya Kong and Jie Ni

Department of General Surgery, Hangzhou First People's Hospital, Nanjing Medical University Affiliated Hangzhou Hospital, PR China

*Corresponding author: Dr. Jia Zhong, Department of General Surgery, Hangzhou First People's Hospital, Nanjing Medical University Affiliated Hangzhou Hospital, PR China,

Tel: +86 13958114181; Fax: +86-0571-87914773; E-mail: jiazhong20058@hotmail.com

Received date: April 06, 2017; Accepted date: April 17, 2017; Published date: April 24, 2017

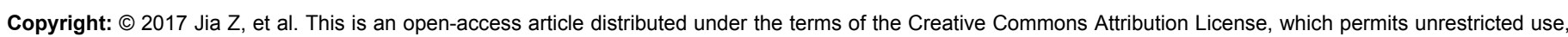
distribution, and reproduction in any medium, provided the original author and source are credited.

\begin{abstract}
Ascitic hernia is still a thorny task but sometimes it may severely influence patients to live a simple but quality or functional life. Herein, we discuss regarding a 37-year-old man with a basketball like indirect inguinal ascitic hernia who was bedridden due to large volume of cirrhotic ascites. All conservative therapies seemed to be only little efficient, only surgery could save him. Based on our past decade's successful experience, where we used to apply an improved technique (Hernia-Roof/Hernia-Sac-Top (HST) approach) to solve scrotum ascitic hernia, aiming to strengthen resistant degree of groin area with little damage to its natural structure. Our technique has been confirmed a practical method in China, and reported in many Chinese version of journals. The technique has three advantages below:
\end{abstract}

1) Ascites was preoperatively managed to ensure the incision wound.

2) The hernia orifice was entirely exposed and closed with suture completely for the first time.

The hernia sac itself, the lower mesh and the orifice-closed were fixed together via 3-D skills. The patient stood up again like a true man. Now he has been followed up in outpatient doctor's office since 10 years, No complications were found. After that, more cases including emergency cases were successfully performed. It is inspiring and has educational value for both patients and surgeons.

Keywords: Ascites; Scrotum hernia; Drainage catheter

\section{Clinical Problem}

A 37-year-old man who was indeed deprived of walking or even taking on his own short trousers just due to his basketball like indirect inguinal hernia with huge volume of progressive hepatitis B-related cirrhotic ascites. He had hepatitis B-related cirrhosis for nearly 20-year history but about 1 year prior to his admission in our hospital he was bedridden due to his huge inguinal ascitic hernia. More awfully, no surgeon was ready to take up his case; despite that he had visited many major hospitals in China. He gradually fell into depression and committed suicide as many doctors told him that the case like him was intractable and thorny, because surgical repair would face high-risk of postoperative complications such as hernia recurrence, bulging of the repaired area, blood leaking, scrotum edema or incision effusion resulting from high pressure of ascites and liver function detrition incoherent. It also means failure of surgery ultimately.

\section{Strategies}

As we all known, there are many causes, including cirrhosis [1], malignancy [2], self-immuno deficiency, etc., may lead to ascitic hernia (umbilical hernia and/or groin hernia), but we sometimes have to admit the fact that some etiologies of ascites cannot be removed or rooted out at all. No surgeon would accept the provisional failure to lower his/her fame. At first, we tried our best to reduce ascites through comprehensive therapies (protection of liver function, albumin supply and diuresis, etc.) to control volume of ascites, however all conventional therapies seemed to take no effects which was discouraging both the patient and physicians. We planned to give up surgery for the patient after a month's concerted-efforts but the patient told us that he preferred dying if doctors couldn't let him stand up like a man, and his families bended their knees before us. We have no choice but just facing the big challenge.

To the best of our knowledge, the key to success is based two aspects:

\section{To downsize volume of ascites.}

To strengthen groin area sustainable performance via hernia repair with little damage to its nature anatomic structure.

Hence, we thought of placement of peritoneal dialysis catheter to drain abdominal ascites during perioperative therapeutic course, aiming to promise decompression of pressure [1]. The dialysis tube has 20-30 side pores in its top end so as to ensure drainage adequately, in addition, the tube is made of silicon material, which is not easy to adhese to the adjacent tissues. All written protocols consent from the patient and his family as well as ethnic committee of our institution were signed. At first, the drainage tube was placed just in the general ward under local anesthesia [1]. Now for the sake of safety, it can be placed under ultrasound-guided percutaneous drainage catheter. The dialysis tube can also be instated with $16-20^{\circ} \mathrm{F}$ of drainage tube as the alternative choice. We found that placement of drainage tube(s) during surgery was found to be more convenient and practical. 


\section{Solving Surgery}

Based on our experience, we adopted preperitoneal approach of groin hernia repair via the hernia-roof/hernia-sac-top (HST) access, which can ensure the integrity of the ventral transverse fascia, simultaneously, it can also enhancing its high-degree of resistant ability to support pressure of ascites.

Which protocol or surgical approach will be accessible and feasible? Whichever approach will benefit the patient the most? Generally, laparoscopic approach is not suitable for this case with fluid-filled abdominal cavity [2]. It may lead to hepatic coma if we release all ascites to gain a clear vision of operative field. In addition, the postoperative growing ascites produced more possibility of progressive pressure and potential of trocar hernia formation. Hence, endoscopic techniques were not considered at all. Herein, we conducted a wisdom procedures below $[3,4]$.

We used a soft, light-weight, flat mesh (including upper mesh and lower mesh) for inguinal hernia repair via the HST/henia-roof technique. Few ascites-drained and a week later, the operation was performed under general anesthesia with the patient in the supine position. Through an incision of about $3-4 \mathrm{~cm}$, the skin, subcutaneous tissues, and aponeurosis of the external oblique muscles were open to expose the hernia-roof.

The hernia sac was promptly addressed when the cremaster muscle opened, and then the outside cover of true hernia is open. As a matter of fact, the outside cover here consists of superficial layer of the transversalis fascia and then, we sutured the hernia-roof with an absorbable thread as an indicator in avoidance of the hernia sac sliding back through the hernia orifice.

The hernia-roof is opened, there is no necrous intestinal or other tissue found except for a little ascites. And then, a small incision is made to the opposite lower quadrant to replace an abdominal drainage tube in the lowest point of pelvis, the procedure of placement of drainage tube can be guided easily if the operator's indicator finger stretch into abdominal cavity through the open access of hernia. After that the operator's finger spreads into inside of hernia as a support so as to promise entire hernia sac stripped off easily [3]. After that, the open access of hernia is closed continuously with barbed suture. Now we strip off all outside cover carefully until the hernia orifice (the peritoneal anterior ring) is exposed completely. In fact, the hernia orifice consists of the deep layer of transversalis fascia. The size of hernia orifice in diameter is nearly $5 \mathrm{~cm}$. The edge of hernia orifice became thickened and flexible. We think that the protection of the edge from damage and closure of hernia orifice is the crucial key to maintain high-degree support of groin area. After that, we fixed the lower flat mesh (Tianchangwuyun Ltd. China) on the top area of hernia sac with sutures. The operator gripped the leash of the lower mesh with an oval clamp, and then pushes both hernia sac and the lower mesh together into preperitoneal space through the hernia orifice to push the flat mesh and hernia sac it into the preperitoneal space, and then the lower mesh was fully flattened with the operator's finger. After that, we pulled out the leash so as to let the mesh closer to the hernia orifice, then, we closed the hernia orifice and the opened superficial transversalis fascia with continuous suture, meanwhile, the bottom of the leash was fixed on the thickened edge of the orifice. After that, the excessive leash was tripped out. As a result, the operative field of groin area looks nature but stronger, because the hernia sac, the lower mesh and the hernia orifice-closed had been tied into an unremovable unity [4]. c). The upper mesh was placed as previous report [3,4], aiming to further enhance the supportive power of the groin area. The aponeurosis of external oblique muscles was sutured continuously. Before the procedure, another drainage tube connected with a negative pressure suction device was placed under the aponeurosis of external oblique muscle, and the top end of drainage tube was also placed just at the lowest point of groin area.

Although the surgery was pretty perfect, the patient's liver function and blood-clot function went worse at first week after surgery due to impact of the surgical trauma and drugs of general anesthesia. At the same time, the incision wound found mild bloody fluid effusion continuously. Amazingly, the protogen time (PT) was obviously delayed even beyond the upper limit of laboratory machine test. We packed the wound with gauze and bandage beside administration of blood-stop drugs. A week later, the wound effusion gradually disappeared, so we drew out the abdominal drainage tube timely. Another drainage tube under the wound was also drawn out when no fluid was drained for 3 days (at postoperative day 9). The wound suture was removed at postoperative day 21 . The patient recovered uneventfully, he finally stood up again as all expected. Now he has been under regular checkups since 10 -years follow-up at outpatient office. No any surgery-related complications such as hernia recurrence were found.

\section{Discussion}

In the latest decade, we have succeeded in performing similar cases (i.e., umbilical ascytic hernia, different inguinal ascytic hernia) to nearly 20 patients. We believe these successful cases confirmed the approach is worthy of learning.

We adopted peritoneal dialysis catheter to release abdominal pressure perioperatively, aiming to ensure the safety and success of the surgery. However, we sometimes encountered cases with carcinoted umbilical hernia and/or groin hernia, at this moment we have no enough time to place the drainage tube before emergency surgery. Based on our experience, the drainage tube can be placed well or readjusted during surgery. Recently the sporadic case reported sublayed hernia repair to solve the dilemma [5].

As we all known Bassini [6] first time described inguinal hernioplasty in the 1880 s, aiming to narrowing the hernia orifice. Shouldice technique stressed on enhancing posterior wall of inguinal hernia via overlapping suture of fasias. Nowadays, tension-free hernia repair has become major prevalence in the world. In western countries, Likinstein technique is major prevalence aiming to block the hernia orifice by pining a mesh on it. While in eastern countries, we prefer to choose preperitoneal approach for groin hernia repair, aiming to hedge a mesh between all hernia orifices and peritoneum. In 2010, surgeons [7] found the preperitoneal ring in inguinal hernia repair by the laparoscopic totally extraperitoneal technique. Obviously the preperitoneal loop is just the hernia orifice. Based on these evidences, the hernia orifice had better be closed completely if you plan to further enhance the support strength of groin area. Although we performed open surgery for cirrhotic patients with ascitic hernia for about less than 20 cases, the satisfactory outcomes have confirmed the technique's efficiency.

Notely, whether the distal sacroma hernia stripped off or not is still full of controversial. In my opinion, the scaroma hernia sac can be easily stripped off as long as you separate it along the right gap between the superficial and deep layer of the ventral transverse fascia, if so, 
Citation: Jia Z, Zhou Y, Kong S, Ni J (2017) Scrotum Hernia with Large Volume of Ascites. J Gen Pract (Los Angel) 5: 303 . doi: 10.4172/2329-9126.1000303

Page 3 of 3

scrotum hematoma or scrotum edema or scrotum fluid accumulation can be reduced or avoided.

At first, we feared of the incision wound which was severely influenced by blood leaking of tiny vessels and/or ascites fistula. So it's essential to place double drainage tubes (one to decompress the inner pressure of abdomen, another one to drain postoperative fluid accumulated under the incision wound) just like a work under the water. The temporary drainage wins enough time to allow the incision cured in schedule.

The improved technique for cirrhotic patients with ascitic inguinal hernia was reported in China (Chinese version of journals) (7-10). Herein, I would like to share my story to more physicians and hope the technique gets attention.

The innovative and unique technique has aroused many hernia specialists' interesting as well as some of worthy debatable issues. For example, some experts thought that the technique was just an improved preperitoneal approach. Some experts ever suspected it's rational. Authors in this study have tried to clarify their technique again and again. Only act in clinical practice will see its truth, consequently authors' technique really does matter to better management of ascitic hernia.

\section{Conclusions}

Hernia-roof/HST approaches along with better management of perioperative ascites may be of crucial importance to effective treatment of inguilar ascitic hernia or seminal cases. The key to the unique technique is to integrate the true hernia, the lower mesh and the hernia inner orifice-closed into an unrecoverable unit by suture.

\section{Authors Contributions}

Dr. Jia Zhong put Hernia-Roof/Hernia-Sac-Top (HST) approach into clinical practice, aiming to strengthen resistant degree of groin area and better management of perioperative drainage of ascites. My student Kong Siya helped me edit the manuscript. My student Zhou Yu helped me update the referred literature. Dr. Ni Jie helped me submit the manuscript.

\section{Written informed consent}

All informed consent about the improved technique and medical data has been obtained from the patient and his family.

\section{Acknowledgement}

The study was sponsored by Hangzhou Science and Technology Program of Zhejiang Province (No: 20150733004)

\section{References}

1. Jia Z, He GH, Feng GH (2005) Surgical therapy of inguinal hernia in cirrhotic patient with refractory ascites. Bull Med Res (Chinese) 34: 23-24.

2. Cavazzola LT, Rosen MJ (2013) Laparoscopic versus open inguinal hernia repair. Surg Clin North Am 93: 69-79.

3. Jia Z, Wu K, Kong SY (2016) Optimized techniques may avoid groin bulging after huge hernioplasty. J Med Imp Surg 1: 109.

4. Wu K, Jia Z, Zhu YH (2016) 3-D Herniorrhaphy may be Accessible via Hernia Sac Top Pathway. Surgery Curr Res 6: 272.

5. Fitzgibbons RJ Jr, Greenburg AG (2002) Nyhus and Condon's hernia. (5th edn.). Lippincott William \&Willkins, Philadelphia, Pennsylvania. pp: 173-180.

6. Bassini E (1887) New Spec for the radical cure of hernia Attidel. Italian Med Ass Congress 2: 179-182.

7. Amato B, Moja L, Panico S, Persico G, Rispoli C, et al. (2012) Shouldice technique versus other open techniques for inguinal hernia repair. Cochrane Database Syst Rev 18: 4.

8. Mainik F, Quast G, Flade-Kuthe R, Kuthe A, Schroedl F (2010) The preperitoneal loop in inguinal hernia repair following the totally extraperitoneal technique. Hernia 14: 361-367.

9. Jia Z, Wang P, Zhu YH (2015) A novel preperitoneal approach for tension-free inguinal herniorrhaphy: Hernia Sac Top pathway, Chin J Hernia Abdominal Wall Surg (Chinese) 9: 57-58.

10. Wu K, Jia Z, Jin HC (2016) Anatomical basis and clinical application of preperitoneal tension-free hernia repair via HST approach. Chin J Hernia Abdominal Wall Surg (Chinese) 10: 369-371. 